\title{
Modeling of rigidity dependent CORSIKA simulations for GRAPES-3
}

\author{
B. Hariharan • S.R. Dugad • \\ S.K. Gupta · Y. Hayashi · S.S.R. \\ Inbanathan - P. Jagadeesan - A. Jain • \\ S. Kawakami · P.K. Mohanty • B.S. Rao
}

Received: date / Accepted: date

\begin{abstract}
The GRAPES-3 muon telescope located in Ooty, India records $4 \times 10^{9}$ muons daily. These muons are produced by interaction of primary cosmic rays (PCRs) in the atmosphere. The high statistics of muons enables GRAPES-3 to make precise measurement of various sun-induced phenomenon including coronal mass ejections (CME), Forbush decreases, geomagnetic storms (GMS) and atmosphere acceleration during the overhead passage of thunderclouds. However, the understanding and interpretation of observed data requires Monte Carlo (MC) simulation of PCRs and subsequent development of showers in the atmosphere. CORSIKA is a standard MC simulation code widely used for this purpose. However, these simulations are time consuming as large number of interactions and decays need to be taken into account at various stages of shower development from top of the atmosphere down to ground level. Therefore, computing resources become an important consideration particularly when billion of PCRs need to be simulated to match the high statistical accuracy of the data. During the GRAPES-3 simulations, it was observed that over $60 \%$ of simulated events don't really reach the Earth's atmosphere. The geomagnetic field (GMF) creates a threshold to PCRs called

* Corresponding authors

B. Hariharan* · S.R. Dugad · S.K. Gupta · P. Jagadeesan · A. Jain · P.K. Mohanty · B.S. Rao

Tata Institute of Fundamental Research, Dr Homi Bhabha Road, Mumbai 400005, India E-mail: 89hariharan@gmail.com

B. Hariharan* · S.R. Dugad · S.K. Gupta · P. Jagadeesan · A. Jain · P.K. Mohanty · B.S. Rao · Y. Hayashi · S. Kawakami

The GRAPES-3 Experiment, Cosmic Ray Laboratory, Raj Bhavan, Ooty 643001, India

B. Hariharan* . S.S.R. Inbanathan*

The American College, Madurai 625002, India

E-mail: ssrinbanathan@gmail.com

Y. Hayashi · S. Kawakami

Graduate School of Science, Osaka City University, 558-8585 Osaka, Japan
\end{abstract}


cutoff rigidity $\mathrm{R}_{c}$, a direction dependent parameter below which PCRs can't reach the Earth's atmosphere. However, in CORSIKA there is no provision to set a direction dependent threshold. We have devised an efficient method that has taken into account of this $\mathrm{R}_{c}$ dependence. A reduction by a factor $\sim 3$ in simulation time and $\sim 2$ in output data size was achieved for GRAPES-3 simulations. This has been incorporated in CORSIKA version v75600 onwards. Detailed implementation of this along the potential benefits are discussed in this work.

Keywords Cosmic Rays · Geomagnetic field · Rigidity · CORSIKA

\section{Introduction}

PCRs are predominantly the nuclei of hydrogen ( $\sim 90 \%)$, helium $(\sim 9 \%)$, and a small fraction $(\sim 1 \%)$ of remaining heavier elements including carbon, nitrogen, oxygen, aluminium, iron etc. They span an energy range from sub-GeV to $10^{11} \mathrm{GeV}$. The PCR flux steeply decreases with energy exhibiting a power law spectrum with a spectral slope of -2.7 . The bulk of the PCRs $(>99.99 \%)$ lie below $100 \mathrm{GeV}$ and are sensitive to inter-planetary magnetic field and solar wind. Thus, the solar activity modulates the PCR flux at these energies. The PCR modulation can be used to study both transient solar phenomenon such as CME, GMS and long-term solar phenomenon related to seasonal, 11-, and 22-year solar cycle over the past eight decades.

The GMF acts as a shield by deflecting out low energy PCRs. Below a certain rigidity value, the PCRs do not reach the Earth's atmosphere. This threshold is known as the geomagnetic cutoff rigidity $\left(\mathrm{R}_{c}\right)$. Since the strength of the GMF varies over the Earth, the $R_{c}$ is also strongly dependent on the location on the Earth. $\mathrm{R}_{c}$ is almost zero at the geomagnetic poles and is about $15 \mathrm{GV}$ at the geomagnetic equator. Although the PCR distribution is known to be almost isotropic, however, the PCR flux observed at the top of the atmosphere is anisotropic due to the variation of $R_{c}$. The PCRs above a few hundred $\mathrm{GeV}$ are least affected by the GMF.

PCRs after entering into Earth's atmosphere interact with the air nuclei and produce secondary particles. These secondary particles further interact down in the atmosphere and produce shower of particles including $\gamma$-rays, electrons, muons and hadrons at the ground level. This process is called cascade shower or extensive air shower (EAS). Unlike other particles, muons due to their energy loss primarily by ionization mostly survive to the ground level. Thus, the muon flux represents a good proxy of the PCR flux. The flux variation in the PCR caused due to various phenomenon as mentioned above could be studied through the measurement of muon flux. In addition, muon flux is also modulated by the atmospheric parameters such as pressure and temperature $[1,2]$. Study of the muon flux variation during thunderstorms has emerged as an exciting area [3].

Interpretation of experimental data requires detailed modeling of EAS development in the atmosphere. This can be studied with standard MC simula- 
tion packages like CORSIKA [4], CRY [5], AIRES [6]. Since these codes have to track large number of particles while taking into account of their hadronic and electro-magnetic interactions, decay processes etc. from the top of the atmosphere to the ground level, the simulations become CPU intensive. The simulation time as well as the generated data size increases linearly with the energy of the PCR. Although the simulation time for low energy PCRs in the $\mathrm{GeV}$ energies is not large, however, it becomes an important consideration when billions of events are required to be simulated to match the high statistical accuracy of data. Even computing farms with over thousands of CPU cores have to be engaged over months to simulate a single physics event $[3,7]$. Thus, any attempt to reduce the simulation time as well as output data size is useful.

CORSIKA simulates PCRs selected at random in a given energy range and spectral slope provided through a control file by the user. The direction of PCRs are chosen randomly from a range of zenith $\theta$ and azimuthal $\phi$ angles which have to be set in the same control file. Normally the minima of the energy range is usually kept well below the lowest $\mathrm{R}_{c}$ for the experiment's location. However, as discussed before, the $\mathrm{R}_{c}$ is not same for all the directions. There was no provision in the earlier versions of CORSIKA to set minimum direction dependent energy in the control file. Thus, in post-simulation, if the PCR's rigidity value is below the $R_{c}$ value of its direction, it had to be rejected since it can not make to the Earth in reality. Thus a significant amount of computing resources had to be unnecessarily wasted. In this work, we discuss a method that avoids simulating events which are below the $\mathrm{R}_{c}$ of respective direction by modifying the CORSIKA code of version v74000. The initial development was reported to the cosmic ray community [8]. By considering its potential benefits, it was recognized by authors of CORSIKA and was made available to the users from CORSIKA version v75600 onwards since 2017. The details of the implementation in CORSIKA with the potential benefits are discussed in following sections.

\section{GRAPES-3 muon telescope}

The GRAPES-3 experiment consists of a large area $\left(560 \mathrm{~m}^{2}\right)$ tracking muon telescope (G3MT) is operating at Ooty in India $\left(11.4^{\circ} \mathrm{N}, 76.7^{\circ} \mathrm{E}, 2200 \mathrm{~m}\right.$ above mean sea level) in conjunction with an array of 400 plastic scintillator detectors as a part of EAS experiment [9]. The G3MT is designed to (1) obtain nuclear composition of PCRs, (2) discriminate $\gamma$-rays from the overwhelming background of charged PCRs, and (3) probe various solar and atmospheric phenomenon. The scintillator detectors are placed in a hexagonal geometry with an inter-detector separation of $8 \mathrm{~m}$. The total area covered by the array is $25000 \mathrm{~m}^{2}$. The EAS array records about $3 \times 10^{6}$ events per day in the energy range of $10^{12}-10^{16} \mathrm{eV}$.

The G3MT uses proportional counter (PRC) as basic detector. Each PRC is a $600 \mathrm{~cm}$ long, $10 \mathrm{~cm} \times 10 \mathrm{~cm}$ cross section mild steel tube with a wall thickness 


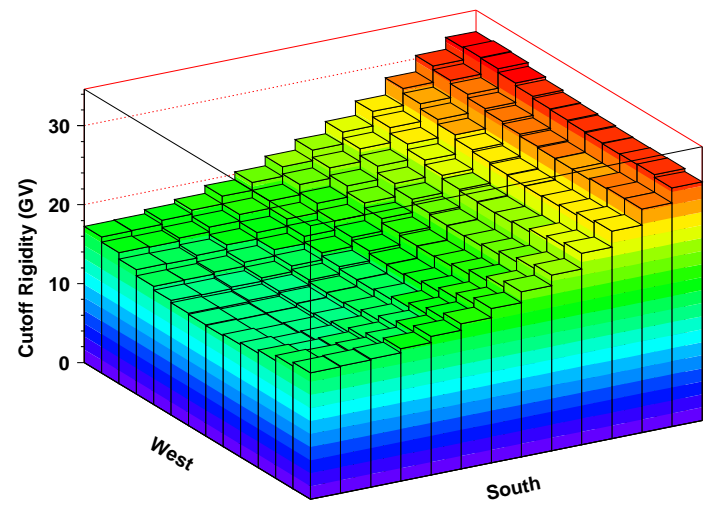

Fig. 1: Map of $\mathrm{R}_{c}$ in the field of view (FOV) of G3MT

of $2.3 \mathrm{~mm}$. A G3MT module consists of 232 PRCs arranged in 4 layers, with alternate layers placed in mutually orthogonal directions which provides an area of $35 \mathrm{~m}^{2}$. Two successive layers of PRCs are separated by $15 \mathrm{~cm}$ thick concrete. The G3MT permits a two-dimensional reconstruction of muon tracks in two vertical orthogonal planes. The vertical separation of two layers of PRCs in the same plane is $\sim 50 \mathrm{~cm}$ which allows the muon track direction to be measured to an accuracy of $\sim 4^{\circ}$. The G3MT permits a two-dimensional reconstruction of muons in 169 directions with the sky coverage of $2.3 \mathrm{sr}$. To achieve an energy threshold of $1 \mathrm{GeV}$ for vertical muons, a total thickness $\sim 550 \mathrm{~g} \mathrm{~cm}^{-2}$ in the form of concrete blocks of $2.4 \mathrm{~m}$ thickness is used as absorber. The concrete blocks have been arranged in the shape of an inverted pyramid to achieve an energy threshold of $\sec (\theta) \mathrm{GeV}$ for muons incident at zenith angle $\theta$ (with coverage up to $45^{\circ}$ ). Four muon modules housed in a single hall to form a super-module and four super-modules constitute the G3MT. It collects $4 \times 10^{9}$ muons daily which has been successfully corrected for efficiency and atmospheric variations [10,2]. The muons recorded by G3MT are produced by PCRs in the energy range of $10^{10}-10^{13} \mathrm{eV}$.

\section{Computation of $\mathbf{R}_{c}$}

The $\mathrm{R}_{c}$ for a given location can be computed numerically using back-tracing method [11]. An anti-proton of a given rigidity is launched from the observer's location into space and traced in the presence of GMF up to several Earth radii. The $\mathrm{X}, \mathrm{Y}$, and $\mathrm{Z}$ components of GMF are evaluated using IGRF-11 coefficients [12]. The trajectory calculation is performed for different rigidities of anti-proton starting from an initial value in decreasing steps of $0.01 \mathrm{GV}$. The rigidity at which the anti-proton trajectory reverses to the Earth is accepted as $\mathrm{R}_{c}$ of that direction. This means a proton of same rigidity coming from outside the magnetosphere will be deflected back into space. $\mathrm{R}_{c}$ is calculated for a grid of $1^{\circ} \times 1^{\circ}$ in zenith $\theta$ and azimuthal $\phi$ angles ranging $0-60^{\circ}$ and 
0-360 ${ }^{\circ}$ respectively. Fig. 1 shows a map of $\mathrm{R}_{c}$ for 169 directions in FOV of G3MT, varies in the range of $12-38 \mathrm{GV}$.

\section{Implementation of $\mathbf{R}_{c}$ in CORSIKA}

CORSIKA is a detailed MC simulation package, developed by KIT, Germany to study the EAS development in the Earth's atmosphere in the energy range of $10^{9}-10^{20} \mathrm{eV}$ for various primaries. Main CORSIKA code contains about 80000 lines written in FORTRAN, with a few optional subroutines in $\mathrm{C}++$. It is interfaced with various external hadronic interaction models such as EPOS, EPOS-LHC[13], QGSJET01C[14], QGSJETII-04[15], SIBYLL[16], VENUS[17], DPMJET[18], NEXUS[19] for high energies (calculation of hadron cross-sections above $80 \mathrm{GeV}$ ) and GHEISHA[20], FLUKA[21], UrQMD[22] for low energies. An atmospheric model and multiple observational levels can be specified in the control file by the user. The secondary particles are tracked till their kinetic energy is above the threshold defined by the user separately for $\gamma$-rays, electrons, muons, and hadrons. The CORSIKA records physical quantities like position, momentum and arrival time of secondary particles up to 10 different observational levels.

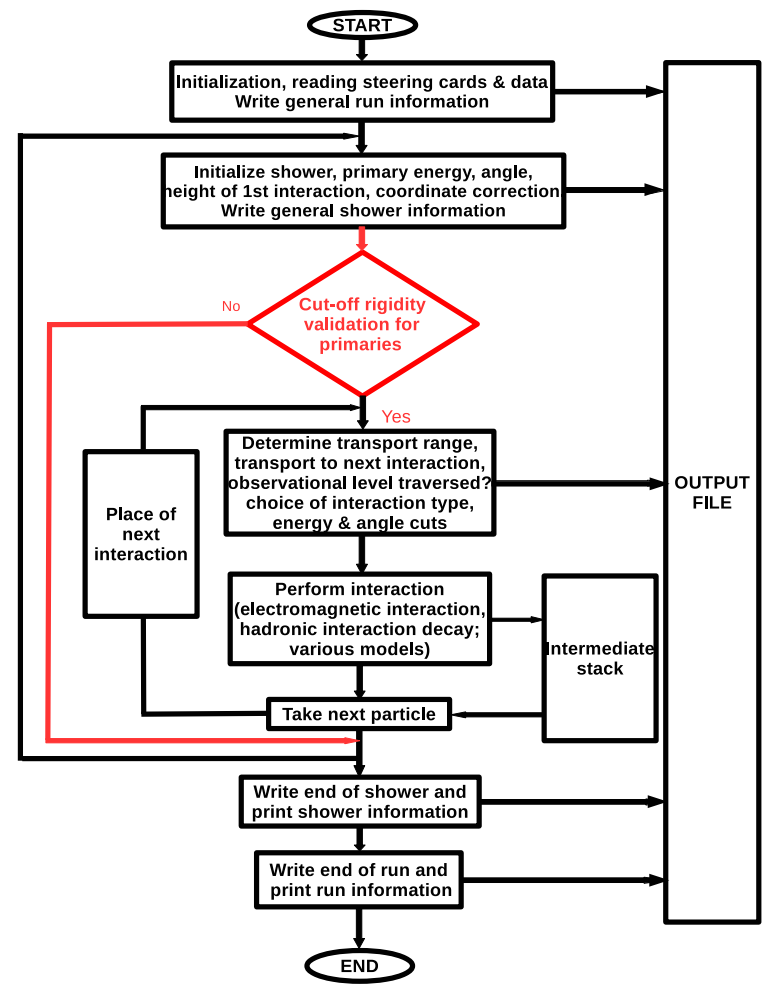

Fig. 2: CORSIKA simulation flow (Modification) 
As discussed in the previous section, a database of $\mathrm{R}_{c}$ is generated for the FOV of G3MT using back-tracing method at a resolution of $1^{\circ} \times 1^{\circ}$ in zenith $\theta$ and azimuthal $\phi$ angles ranging $0-60^{\circ}$ and $0-360^{\circ}$ respectively. Since the computation of $\mathrm{R}_{c}$ is a CPU intensive process, it is difficult to obtain $\mathrm{R}_{c}$ for every PCR in real-time during simulation. Thus an off-line database is made mandatory for this implementation and it has to be calculated once by the users for their observational location. The coordinate convention used in the back-tracking program is astronomical standard where $\phi$ moves clockwise from north. Since in CORSIKA, $\phi$ moves counter-clock wise from the north, thus $180^{\circ}$ rotation is required in database. A simple flow chart of CORSIKA simulation is displayed in Fig. 2 along with modified flow explaining the $\mathrm{R}_{c}$ validation. During initialization of CORSIKA simulation, the $\mathrm{R}_{c}$ database is loaded into CORSIKA. Based on the user inputs, energy and direction of a PCR event is determined at random. Next, a $R_{c}$ value is determined from the map based on the direction $(\theta, \phi)$ of the PCR through a linear interpolation. If the PCR's rigidity is higher than the $\mathrm{R}_{c}$ of that direction, then only further simulation of shower development is allowed otherwise it is rejected. No input and output information of the rejected shower is recorded. As a result, the simulation time and output data size is reduced significantly. Since the composition of PCRs are known to be predominantly proton and helium, the present implementation is made only for proton and helium. However, this can be easily extended for other primaries if required.

\section{Results and discussions}

To test the effectiveness of this method, a small set of simulations were carried out. A total of $10^{8}$ proton primaries were generated with unmodified CORSIKA code over an energy range of $10^{10}-10^{13} \mathrm{eV}$ with a power-law distribution of spectral slope -2.7 in the angular range of $0-60^{\circ}$ and $0-360^{\circ}$ for $\theta$ and $\phi$ respectively (hereafter called case A). The same configuration was repeated with modified CORSIKA code (hereafter called case B). In each of these cases, the simulation was carried out by distributing the total number of events into 1000 jobs in the GRAPES-3 computer cluster having $1280 \mathrm{CPU}$ cores. The energy spectrum of simulated events for both the cases are displayed in Fig. 3. The spectrum (a) in Fig. 3 was generated as per given inputs and falls perfectly in a power-law as expected whereas the spectrum (b) shows only $44 \%$ of input events which underwent the full shower simulation and the remaining $56 \%$ events which were rejected as they did not pass the $\mathrm{R}_{c}$ condition of the respective directions are shown in (c). In case A, by post-simulation processing, $56 \%$ of events were rejected since they are not really useful for analysis even though they were simulated. In Fig. 3, the spectra (a) and (b) are indistinguishable above $35 \mathrm{GeV}$. It is to be noted that $\mathrm{R}_{c}$ validation in CORSIKA are used here with tolerance of $-10 \%$. The tolerance allows the $\mathrm{R}_{c}$ validation to be checked with lower $\mathrm{R}_{c}$ of the primary. This is important for studies where rigidity dependence is crucial. The discovery of transient weakening of Earth's 


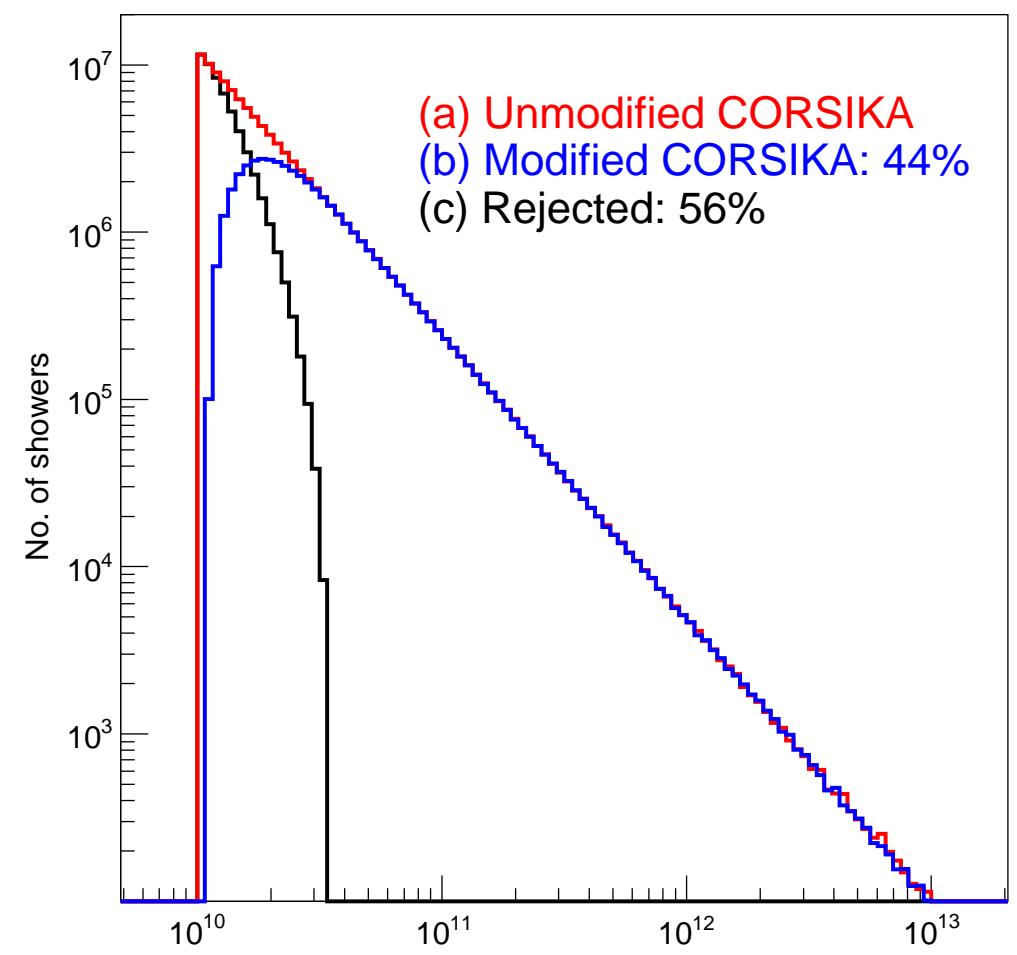

Fig. 3: Energy spectrum (eV) of PCR events simulated by (a) unmodified CORSIKA, and (b) modified CORSIKA. The distribution rejected primaries from modified CORSIKA is shown in (c).

magnetic shield probed by a muon burst is one such example [7]. In this study, the muon burst recorded by G3MT was caused by a G4-class GMS triggered by a CME. The Earth's magnetic shield was weakened due to magnetic reconnection of GMF with magnetic field carried by CME. This lowered the $\mathrm{R}_{c}$ in G3MT vicinity which allowed enormous count of low energy PCRs to enter into Earth's atmosphere resulted in muon burst for 2 hours. The burst was successfully modeled by MC simulations which allowed us to use G3MT as rigidity meter. In such studies to model the rigidity change, it is important to generate CORSIKA events with tolerance, so that repeated simulations can be avoided. One would expect higher rejection $(\sim 63 \%)$, leads to larger reduction in simulation time and data size if $0 \%$ tolerance is given in the validation. However it is recommended to use with small tolerance, so that the repetition of simulations can be avoided in future if $\mathrm{R}_{c}$ has to be recalculated or the same data set has to be used for another EAS experiment.

The real proof of principle for this implementation is demonstrated by comparing physics parameters from both these cases. The simulated datasets from both cases were analysed with in-house developed G3MT detector simulation code. The in-house simulation code simulates every CORSIKA generated muon in detector geometry to find hits in four layers of PRCs. It also takes care of 


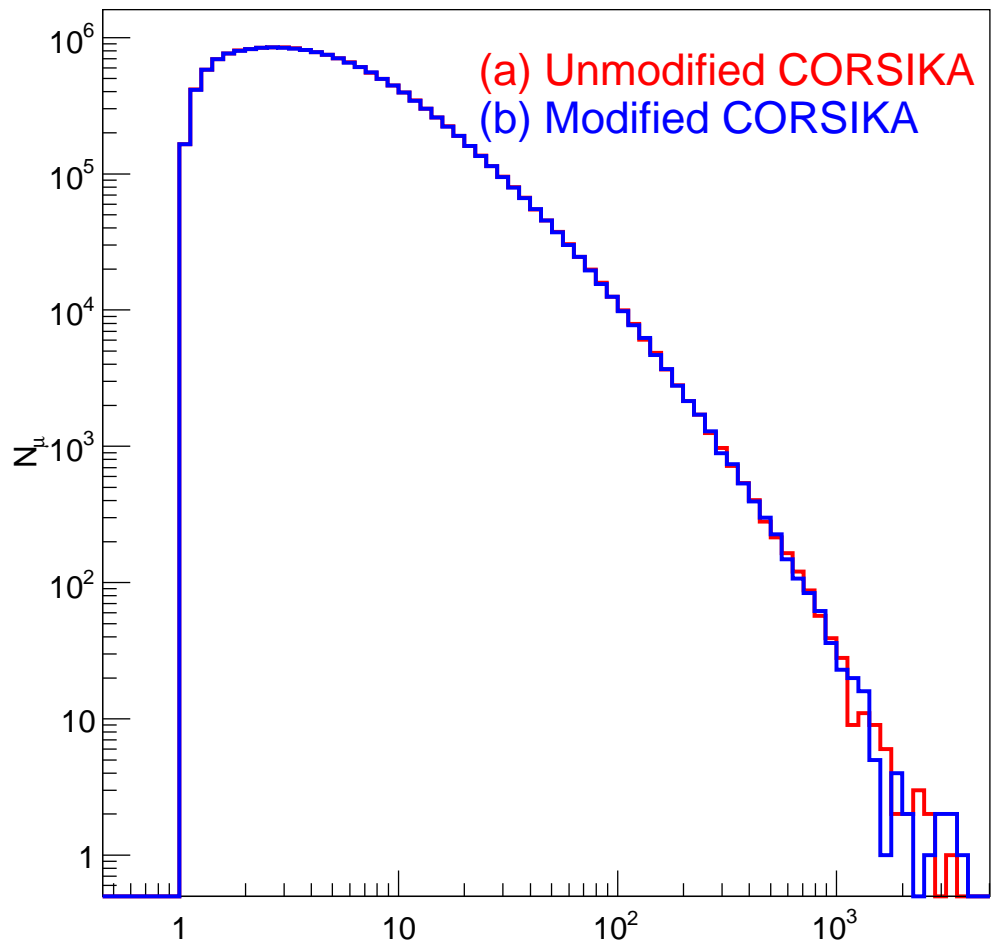

Fig. 4: Muon energy spectrum (GeV) obtained from (a) unmodified CORSIKA (b) modified CORSIKA

angular threshold and trigger criteria to reconstruct the detected muons in 169 directions using hit patterns which can be easily compared with observation. The muon energy spectrum for all directions obtained from both cases are compared in Fig. 4. The muon energy spectra obtained from both cases in Fig. 4 are identical and small perturbations can be seen at higher energies due to small statistics. These perturbations found in primary and muon energy spectra arise due to change in random number sequence in CORSIKA due to rejected PCR. However, these changes are statistically insignificant.

Table. 1 shows quantitative comparison of outputs obtained from both cases. Significant improvements can be seen in terms of reduction in simulation time by a factor of $\sim 3$ and output data size by a factor of $\sim 2$. Despite of large number of PCRs rejected in case B shown in Fig. 3, the muon spectrum from both cases are almost identical as shown in Fig. 4. This allows the user to generate more events in disputed time by imposing $\mathrm{R}_{c}$. For example, a recent result on the measurement of electrical properties for a thunderstorm event observed in G3MT describes the generation of Giga-Volt potential in thunderclouds [3]. The thundercloud parameters were derived with the aid of MC simulations of atmospheric electric field using CORSIKA. These simulations include generation of $10^{6}$ muons for 60 steps of electric field and $10^{7}$ muons for background for each of 169 directions This extensive simulation was carried 
out in GRAPES-3 computer cluster running continuously for two months. It would have taken more than six months and massive output storage costing $\sim 30$ TB to generate the data bank without this utility.

One of the biggest threat to our technologies is solar storms which can disrupt power grids, telecommunications and navigation systems [23]. Some famous historic evidences are 'Halloween event', 'Quebec event', and 'Carrington event' [24]. The solar super storm 'Carrington event' recorded in 1859 caused a black out of telegraphic systems in high latitude regions of North America and Europe. In order to prepare for such solar storms in future, large number of ground based observatories including network of neutron monitor stations were installed globally and collecting data throughout the year to study near Earth phenomenon. However, the understanding of these phenomenon primarily depends on precise measurement and accurate MC simulations dealing large number of PCRs. Because of vast geographical distribution of these observatories, the $R_{c}$ varies from $0 \mathrm{GV}$ at poles to $15 \mathrm{GV}$ at magnetic equator. Despite of large number of PCRs were rejected during simulation, the physics results derived from analysis were unaffected. The present work provides an advancement in simulating low energy PCRs efficiently by reducing simulation time and data size. This modification was reported to authors of CORSIKA which was implemented in official version from v75600 since 2017.

Table 1: Comparison of simulation parameters

\begin{tabular}{|l|l|l|}
\hline Parameter & Case A & Case B \\
\hline Number of simulated showers & $1 \times 10^{8}$ & $0.44 \times 10^{8}$ \\
Simulation time (min) & 156 & 46 \\
File size (GB) & 239 & 114 \\
\hline
\end{tabular}

Acknowledgements We thank Dr. T. Pierog and Dr. D. Heck for implementing this modification in official CORSIKA releases to benefit cosmic ray community. We thank D.B. Arjunan, A. Chandra, V. Jeyakumar, S. Kingston, K. Manjunath, S.D.Morris, S. Murugapandian, P.K. Nayak, S. Pandurangan, B. Rajesh, P.S. Rakshe, K. Ramadass, K. Ramesh, L.V. Reddy, V. Santhoshkumar, M.S. Shareef, C. Shobana, R. Sureshkumar, and M. Zuberi for their assistance in running the GRAPES-3 experiment.

\section{References}

1. P.K. Mohanty et al., Astroparticle Physics 79 (2016) 23-30

2. K.P. Arunbabu et al., Astroparticle Physics 94 (2017) 22-28

3. B. Hariharan et al., Phys. Rev. Lett. 122, 105101 (2019)

4. D. Heck, J. Knapp, J.N. Capdevielle, G. Schatz, and T. Thouw, Report FZKA 6019 (1998), Forschungszentrum Karlsruhe; available from http://www-ik.fzk.de/corsika/physics_description/corsika_phys.html

5. Monte Carlo Simulation of Proton-induced Cosmic-ray Cascades in the Atmosphere, UCRL-TM-229452

6. AIRES: A system for air shower simulations, arXiv:astro-ph/9911331v1 
7. P.K. Mohanty et al. Phys. Rev. Lett. 117, 171101 (2016);

P.K. Mohanty et al. Phys. Rev. D 97, 082001 (2018)

8. B. Hariharan et al., Proceedings of Science PoS(ICRC2015)448

9. S.K. Gupta et al. Nucl. Instrum. Methods A 540, 311 (2005);

Y. Hayashi et al. Nucl. Instrum. Methods A 545, 643 (2005)

10. P.K. Mohanty et al., Proceedings of Science PoS(ICRC2017)357

11. D. F. Smart and M. A. Shea, Adv. Space Res. 36, 2012 (2005)

12. C. Finlay et al., Geophys. J. Int. 183, 1216 (2010)

13. T. Pierog et al., arXiv:1306.0121[hep-ph] (2013)

14. N.N. Kalmykov, S.S. Ostapchenko, and A.I. Pavlov, Nucl. Phys. B (Proc. Suppl.) 52B (1997) 17

15. S.S. Ostapchenko, Phys. Rev. D83 (2011) 014018

16. R. Engel, T.K. Gaisser, P. Lipari, and T. Stanev, Proc. $26^{\text {th }}$ Int. Cosmic Ray Conf., Salt Lake City (USA), 1 (1999) 415; E.-J. Ahn, R. Engel, T.K. Gaisser, P. Lipari, and T. Stanev, Phys. Rev. D80 (2009) 094003

17. K. Werner, Phys. Rep. 232 (1993) 87

18. J. Ranft, Phys. Rev. D51 (1995) 64; arXiv:hep-ph/9911213 and hep-ph/9911232 (1999)

19. H.J. Drescher, M. Hladik, S. Ostapchenko, T. Pierog, and K. Werner, Phys. Rep. 350 (2001) 93 (preprint hep-ph/0007198 (2000))

20. H. Fesefeldt, Report PITHA-85/02 (1985),RWTH Aachen; available from: http://cds.cern.ch/record/162911/files/CM-P00055931.pdf

21. A. Fassò, A. Ferrari, S. Roesler, P.R. Sala, G. Battistoni, F. Cerutti, E. Gadioli, M.V. Garzelli, F. Ballarini, O.Ottolenghi, A. Empl and J. Ranft, The physics models of FLUKA: status and recent developments, Computing in High Energy and Nuclear Physics 2003 Conference (CHEP2003), La Jolla, CA (USA), March 24-28, 2003 (paper MOMT005); eConf C0303241 (2003); arXiv:hep-ph/0306267; http://www.fluka.org/references.html

22. S.A. Bass et al., Prog. Part. Nucl. Phys. 41 (1998) 225; M. Bleicher et al., J. Phys. G: Nucl. Part. Phys. 25 (1999) 1859; http://urqmd.org/

23. https://obamawhitehouse.archives.gov/the-press-office/2016/10/13/executive-ordercoordinating-efforts-prepare-nation-space-weather-events

24. Severe Space Weather EventsUnderstanding Societal and Economic Impacts: A Workshop Report (National Academies Press, Washington, 2008) doi:10.17226/12507; http://www.oecd.org/gov/risk/46891645.pdf 